\title{
Immuno-EM in Buffer Using the Atmospheric Scanning Electron Microscope (ASEM)
}

Chikara Sato*, Yuusuke Maruyama, ${ }^{*}$ Tatsuhiko Ebihara, ${ }^{*}$ Kazuhiro Mio, ${ }^{*}$ Sachie Manaka, ${ }^{*}$ Hidetoshi Nishiyama, ${ }^{* *}$ and Mitsuo Suga**

* National Institute of Industrial Science and Technology (AIST), Tsukuba, 305-8566, Japan

** JEOL Ltd., 1-2 Musashino 3-chome, Akishima, Tokyo 196-8558, Japan

Protein complexes are essential for various kinds of physiological functions in our body. Their components dynamically change location by dissociating and associating with other proteins, forming higher-order complexes. To understand their mechanisms, it is necessary to determine their localization and to map the complex formation.

Immuno-EM has played an essential role in the determination of protein distribution at high resolutions. The environmental cell for the EM enabled direct visualization of labeled antibodies in a hydrophilic environment, although the capsule's small sample space limited applicable cells and slowed throughput. To overcome these limitations, we have developed an inverted electron microscope, the ASEM, with an open sample holder (Fig.1) [1]. For this new microscope, a sample dish with a pressure-resistant, 100 $\mathrm{nm}$ SiN film window (ASEM Dish) was developed, which allows the electron beam to be projected from underneath the sample. The SEM is directly correlated with an optical microscope $(\mathrm{OM})$ above the sample. The optical axes of both microscopes are aligned to observe the same area pseudo-simultaneously, with a sample stage that can shift the imaging area two-dimensionally. Aside from the $\mathrm{SiN}$ film window, the ASEM dish is a standard $3 \mathrm{~cm}$, several-ml Petri dish. With various surface coatings, this dish allows the culture of different types of cells in a $\mathrm{CO}_{2}$ incubator, e.g., the primary cell culture of hippocampal neurons (Fig.1). Until now, various coatings, including collagen, fibronectin, laminin and poly-L-lysin, have been successful for the ASEM dish. The range of cell types applicable to this system will be further broadened by an increase in the kinds of substrates available for coating the SiN film.

The open ASEM dish allows efficient immuno-labeling using gold-tagged antibodies; especially washing, staining, and transfection are easy. Because this electron microscope can directly observe cells in buffer, epitopes are well preserved in the hydrophilic environment. Our success rate with various labeling antibodies used for fluorescence optical microscopy was very high with the ClairScope ${ }^{\mathrm{TM}}$, enabling observation at SEM resolutions. For example, using a secondary antibody dually tagged with fluorescence and gold, we succeeded in determining the distribution of the glycan-receptor CD44 protein in glioblastoma U-251 cells [2]. Together with an antibody associated with the target protein, the correlation between OM and EM can visualize co-localization of proteins, suggesting protein-complex formation. Using this dual-labeling, we observed various proteins, such as the cytoskeletal systems in Fig.2.

References

[1] H. Nishiyama et al., J. Struct. Biol. 169 (2010) 438-449.

[2] T. Murai et al., J. Biol. Chem. 286 (2011) 1999-2007. 


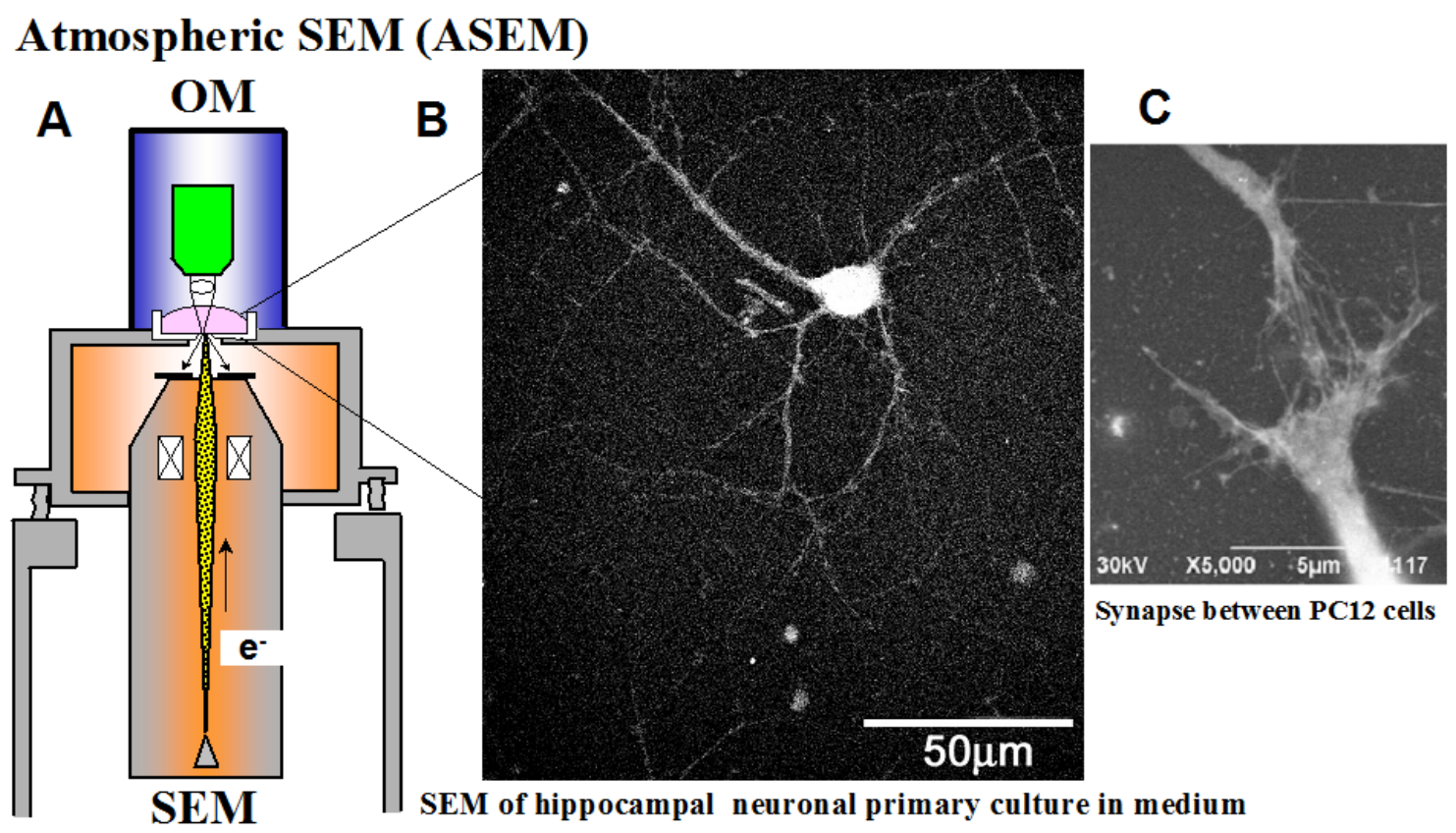

Fig. 1 Configuration of the ASEM; image of neurons. (A) The SEM has a totally inverted structure, with electron gun at the bottom. An optical microscope (OM) is arranged above/opposite the inverted SEM with the specimen dish between. The removable, several ml-capacity dish features a $\mathrm{SiN}$ film window. (B) SEM of primary culture of hippocampal neurons in buffer. (C) SEM of synaptic connections between PC12 cells. We can observe many connections at x5000 magnification.
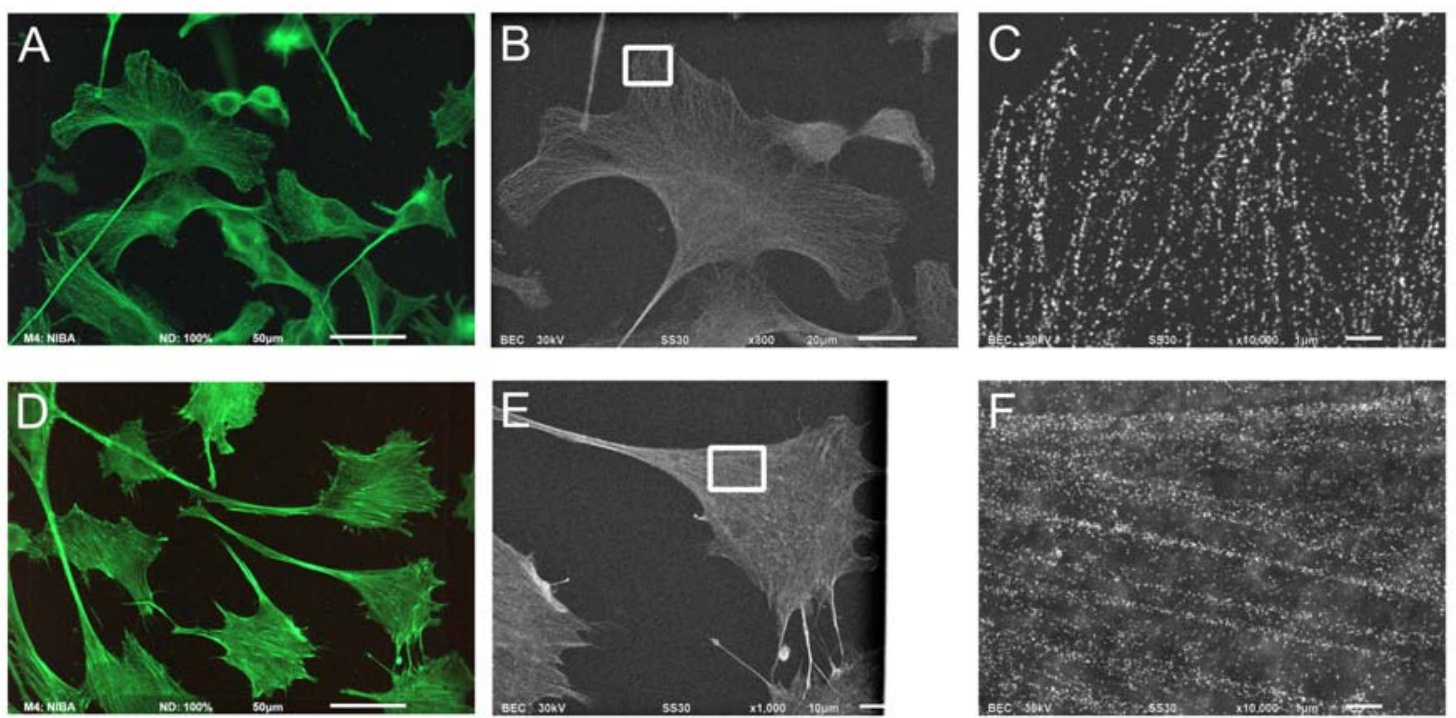

Fig. 2 Immuno-EM of cytoskeleton using the correlative ASEM: SEM and OM. C2C12 muscle progenitor cells fixed with aldehyde are labeled with anti-microtubule antibody, and further tagged with fluorescence and gold. Fluorescence (A) is directly compared with the SEM image in buffer at $x 800$ (B) and at x10000 (C). Affinity-labeled fluorescence microscopy (D) of F-actin is compared with affinity gold-labeled EM at $\mathrm{x} 1000(\mathrm{E})$ and at $\mathrm{x} 10000(\mathrm{~F})$. 